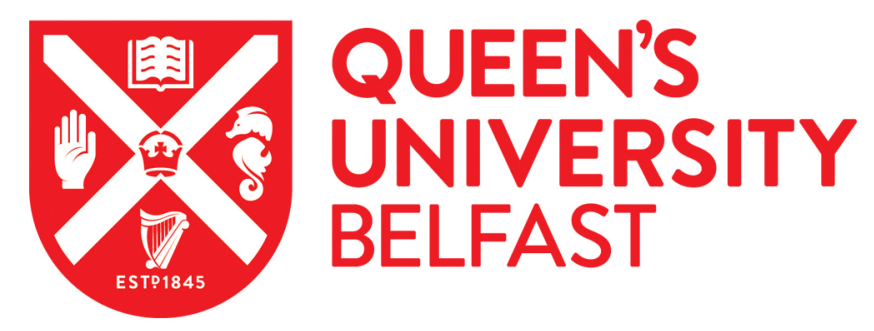

\title{
Kelvin-Helmholtz instability in solar chromospheric jets: theory and observation
}

Kuridze, D., Zaqarashvili, T. V., Henriques, V., Mathioudakis, M., Keenan, F. P., \& Hanslmeier, A. (2016). KelvinHelmholtz instability in solar chromospheric jets: theory and observation. The Astrophysical Journal, 830(2), [133]. https://doi.org/10.3847/0004-637X/830/2/133

\section{Published in:}

The Astrophysical Journal

Document Version:

Publisher's PDF, also known as Version of record

Queen's University Belfast - Research Portal:

Link to publication record in Queen's University Belfast Research Portal

Publisher rights

(c) 2016. The American Astronomical Society. All rights reserved.

This work is made available online in accordance with the publisher's policies.

\section{General rights}

Copyright for the publications made accessible via the Queen's University Belfast Research Portal is retained by the author(s) and / or other copyright owners and it is a condition of accessing these publications that users recognise and abide by the legal requirements associated with these rights.

Take down policy

The Research Portal is Queen's institutional repository that provides access to Queen's research output. Every effort has been made to ensure that content in the Research Portal does not infringe any person's rights, or applicable UK laws. If you discover content in the Research Portal that you believe breaches copyright or violates any law, please contact openaccess@qub.ac.uk. 
KELVIN-HELMHOLTZ INSTABILITY IN SOLAR CHROMOSPHERIC JETS: THEORY AND OBSERVATION

\author{
D. Kuridze ${ }^{1,5}$, T. V. Zaqarashvili ${ }^{2,3,4}$, V. Henriques ${ }^{1}$, M. Mathioudakis ${ }^{1}$, F. P. Keenan ${ }^{1}$, And A. Hanslmeier ${ }^{2}$ \\ ${ }^{1}$ Astrophysics Research Centre, School of Mathematics and Physics, Queen's University, Belfast BT7 1NN, UK; d.kuridze@qub.ac.uk \\ ${ }^{2}$ IGAM, Institute of Physics, University of Graz, Universitätsplatz 5, A-8010 Graz, Austria \\ ${ }^{3}$ Abastumani Astrophysical Observatory at Ilia State University, 3/5 Cholokashvili avenue, 0162 Tbilisi, Georgia \\ ${ }^{4}$ Space Research Institute, Austrian Academy of Sciences, Schmiedlstrasse 6, A-8042 Graz, Austria \\ ${ }^{5}$ Abastumani Astrophysical Observatory at Ilia State University, G. Tsereteli 3, 0162, Tbilisi, Georgia \\ Received 2015 December 3; revised 2016 July 27; accepted 2016 August 4; published 2016 October 18
}

\begin{abstract}
Using data obtained by the high-resolution CRisp Imaging SpectroPolarimeter instrument on the Swedish $1 \mathrm{~m}$ Solar Telescope, we investigate the dynamics and stability of quiet-Sun chromospheric jets observed at the disk center. Small-scale features, such as rapid redshifted and blueshifted excursions, appearing as high-speed jets in the wings of the $\mathrm{H} \alpha$ line, are characterized by short lifetimes and rapid fading without any descending behavior. To study the theoretical aspects of their stability without considering their formation mechanism, we model chromospheric jets as twisted magnetic flux tubes moving along their axis, and use the ideal linear incompressible magnetohydrodynamic approximation to derive the governing dispersion equation. Analytical solutions of the dispersion equation indicate that this type of jet is unstable to Kelvin-Helmholtz instability (KHI), with a very short (few seconds) instability growth time at high upflow speeds. The generated vortices and unresolved turbulent flows associated with the KHI could be observed as a broadening of chromospheric spectral lines. Analysis of the $\mathrm{H} \alpha$ line profiles shows that the detected structures have enhanced line widths with respect to the background. We also investigate the stability of a larger-scale $\mathrm{H} \alpha$ jet that was ejected along the line of sight. Vortex-like features, rapidly developing around the jet's boundary, are considered as evidence of the KHI. The analysis of the energy equation in the partially ionized plasma shows that ion-neutral collisions may lead to fast heating of the KH vortices over timescales comparable to the lifetime of chromospheric jets.
\end{abstract}

Key words: magnetohydrodynamics (MHD) - methods: analytical - Sun: atmosphere - Sun: chromosphere - Sun: transition region - techniques: imaging spectroscopy

\section{INTRODUCTION}

The chromosphere is a highly inhomogeneous layer of the solar atmosphere, populated by a wide range of dynamical features such as spicules (type I and II), fibrils, mottles, rapid excursions (REs) which can be redshifted (RRE) or blueshifted (RBE), and surges (see the review by Tsiropoula et al. 2012). These are smallscale, short-lived, jet-like plasma structures observed near the network boundaries ubiquitously between the photosphere and the corona. However, they have different physical properties and evolution cycles. In particular, the traditional (type I) spicules and mottles have lifetimes ranging from 1-12 minutes and are characterized by rising motions with speeds of $\sim 20-40 \mathrm{~km} \mathrm{~s}^{-1}$. At the last stage of their lifetime, type I spicules either fall back to the low chromosphere with a speed comparable to their upflow value, or fade gradually without any descending motion. Zhang et al. (2012) have reported that around 60\% of type I spicules have a complete cycle of ascent and descent movement. There are larger scale $\mathrm{H} \alpha$ jets in the chromosphere such as macrospicules and surges which could have diameter and flow speeds of a few $\mathrm{Mm}$ and $\sim 100 \mathrm{~km} \mathrm{~s}^{-1}$, respectively.

A very prominent class of spicular-type jets are the so-called type II spicules and their on-disk counterparts, RBEs/RREs (de Pontieu et al. 2007; Langangen et al. 2008, hereafter termed REs). REs are absorption features detected in the blue and red wings of chromospheric spectral lines (Langangen et al. 2008; Rouppe van der Voort et al. 2009; Sekse et al. 2013b; Kuridze et al. 2015a). They are slender ( $200 \mathrm{~km}$ in width), short-lived ( $\sim 0 \mathrm{~s})$ with higher apparent velocities of $\sim 50-150 \mathrm{~km} \mathrm{~s}^{-1}$.

The formation mechanism of spicules is not well understood, with several possibilities proposed, including: magnetic reconnection (Uchida 1969; Pataraya et al. 1990), granular buffeting (Roberts 1979), velocity pulses (Suematsu et al. 1982), rebound shocks (Hollweg 1982; Murawski \& Zaqarashvili 2010), Alfvén waves (Hollweg 1981), and p-mode leakage (De Pontieu et al. 2004). These models can explain the formation of classical spicules to some extent, but the formation of REs/type II spicules with their extremely high upflow speeds $\left(\sim 50-150 \mathrm{~km} \mathrm{~s}^{-1}\right)$ remains a mystery. Song \& Vasyliūnas $(2011,2014)$ found that the high heating rate in the strong magnetic field region in the upper chromosphere is able to produce type II spicules, based on their model of the strong damping of Alfvén waves via plasmaneutral collisions. Magnetohydrodynamic (MHD) simulations also struggle to reproduce the observational characteristics of spicules, including heights, lifetimes, velocities, densities, and temperatures.

REs are also characterized by rapid fading in the chromospheric spectral line without any descending behavior. It is suggested that the rapid disappearance of the spicular jets may be the result of their fast heating to transition region (TR) temperatures. De Pontieu et al. (2011) provided evidence that TR and coronal brightenings in AIA passbands are occurring co-spatially and co-temporally with chromospheric RBEs. Furthermore, Madjarska et al. (2011) showed that the coronal hole large spicules observed with Hinode in the $\mathrm{Ca}$ II $\mathrm{H}$ line appear in the TR O V $629.76 \AA$ line observed with the SUMER instrument on-board $\mathrm{SOHO}$. The analysis of the differential emission measure distribution for a region dominated by spicules also indicates that they are heated to TR temperatures (Vanninathan et al. 2012). Recently, Pereira et al. (2014) studied the thermal evolution of type II spicules using combined observations from the Hinode and Interface Region 
Imaging Spectrograph (IRIS) satellites. They showed that the fading of spicules from the chromospheric $\mathrm{Ca}$ II $\mathrm{H}$ line is caused by rapid heating of the upward-moving spicular plasma to higher temperatures. More recently, Rouppe van der Voort et al. (2015) found spectral signatures for REs in the IRIS C II 1335 and $1336 \AA$ and Si iv 1394 and $1403 \AA$ spectral lines, and interpreted those as evidence that REs are heated to at least TR temperatures. Furthermore, the IRIS observations revealed the prevalence of fast network jets with lifetimes of 20 to $80 \mathrm{~s}$, widths of $\leqslant 300 \mathrm{~km}$ and upflow speeds of $80-250 \mathrm{~km} \mathrm{~s}^{-1}$, with no downward component (Tian et al. 2014). Spectroscopic observations from IRIS reveal that many of these jets are heated to $\sim 10^{5} \mathrm{~K}$ (Tian et al. 2014). Henriques et al. (2016) found a statistically significant match between automatically detected heating signatures in the corona, as observed in the AIA passbands, and quiet-Sun REs, with a minimum of $6 \%$ of the detections at $\sim 10^{6} \mathrm{~K}$ (AIA Fe IX 171) being attributable to REs.

Despite a wealth of observations, the heating mechanism associated with chromospheric jets remains a mystery. Recent theoretical studies suggest that the Kelvin-Helmholtz instability (KHI) could be a viable mechanism for the observed fast heating of chromospheric jets (Zaqarashvili 2011; Kuridze et al. 2015a; Zaqarashvili et al. 2015). Mass flows in the chromospheric fine structures can create velocity discontinuities between the surface of the jets and surrounding media, which may trigger the KHI in some circumstances depending on the directions of flows and magnetic fields.

The theory of KHI in solar atmospheric events has been intensively developed in recent years. It has been studied in spicular-like chromospheric jets (Zaqarashvili 2011; Zhelyazkov 2012), magnetic tubes with partially ionized plasmas (Soler et al. 2012; Martínez-Gómez et al. 2015) in photospheric tubes (Zhelyazkov \& Zaqarashvili 2012), and twisted and rotating magnetic jets (Zaqarashvili et al. 2010, 2014, 2015). It was shown that the energy conversion mechanism called resonant absorption (Goossens et al. 2002, 2006, 2011) may play an important role in the onset of KHI instability in magnetic flux tubes. I.e., the transverse MHD oscillations in coronal loops can lead to KHI through resonant absorption in a narrow inhomogeneous layer that can deform the crosssectional area of the loops (Ofman et al. 1994; Terradas et al. 2008; Soler et al. 2010; Antolin et al. 2014). Furthermore, it is suggested that the mode conversion from magneto-acoustic oscillations across the whole jet to small-scale localized Alfvén motions due to resonant absorption may also create KHI through phase mixing (Browning \& Priest 1984). As well as the theory, recent observations show the presence of KHI in the solar corona, e.g., in prominences (Berger et al. 2010; Ryutova et al. 2010), coronal mass ejections and helmet streamers (Foullon et al. 2011, 2013; Ofman \& Thompson 2011; Feng et al. 2013; Möstl et al. 2013).

Recently, Kuridze et al. (2015a) estimated the growth rate of KHI in a transversely-moving REs using a simple slab model. They showed that the REs moving in the transverse direction could be unstable due to KHI with a very short $(\sim 5 \mathrm{~s})$ instability growth time.

In this paper we analyze the data presented in Kuridze et al. (2015a) to study the dynamics of $\mathrm{H} \alpha$ jets. We adopt the theoretical model of KHI for twisted magnetic jets in the solar wind developed by Zaqarashvili et al. (2014), and derive a governing dispersion equation to investigate the stability of chromospheric $\mathrm{H} \alpha$ jets. Using the theoretical model and

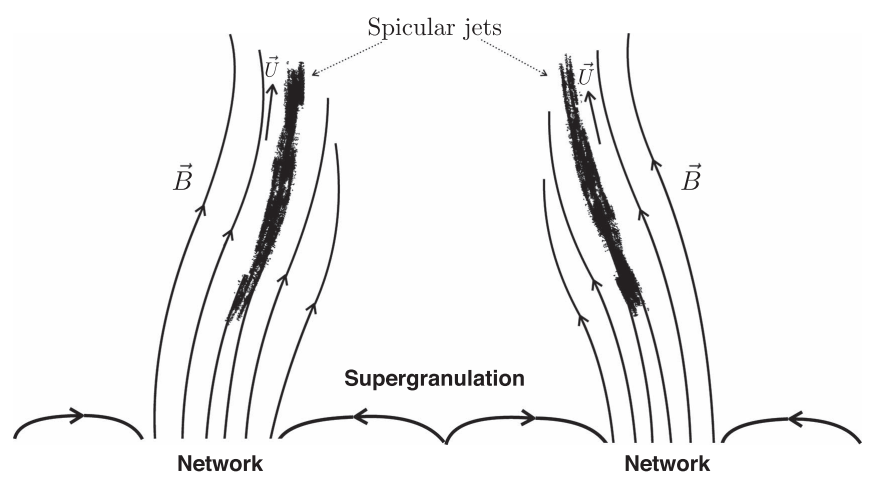

Figure 1. Simple schematic diagram of a spicular jet considered as cylindrical, high-density, twisted magnetic flux tubes moving in an axial direction with respect to a twisted external field.

observed jet parameters we estimate the growth time of the KHI in $\mathrm{H} \alpha$ jets. Furthermore, various energy dissipation mechanisms that could be responsible for the heating of chromospheric jets due to KHI are investigated using the energy equation of the partially ionized plasma. Observational evidence that could be considered as a signature of the KHI in the analyzed structures is also presented and discussed.

\section{KHI: THEORY}

The KHI theory of twisted magnetized jets in the solar wind was developed by Zaqarashvili et al. (2014). They showed that, in the linear incompressible MHD limit, KHI is suppressed in the twisted tube by the external axial magnetic field for subAlfvénic motions. However, even a small twist in the external magnetic field allows KHI to develop for any sub-Alfvénic motion. Based on their approach, in our theoretical model the chromospheric jets are considered as cylindrical, high-density, twisted magnetic flux tubes with radius $a$ embedded in a twisted external field (Figure 1). We use a cylindrical coordinate system $(r, \phi, z)$ and assume that the magnetic field has the following form: $B=\left(0, B_{\phi}(r), B_{\mathrm{z}}(r)\right)$. The unperturbed magnetic field and pressure $\left(p_{0}\right)$ satisfy the pressure balance condition

$$
\frac{d}{d r}\left(p_{0}+\frac{B_{\phi}^{2}+B_{z}^{2}}{8 \pi}\right)=\left(\frac{B_{\phi}^{2}}{4 \pi r}\right) .
$$

The magnetic field inside and outside the jets is considered to be weakly twisted, thus not leading to the kink instability, and the tube moves along the axial direction with regard to the surrounding medium with speed $U$ (Figure 1). In the cylindrical coordinate system the magnetic field inside and outside the tube is $\boldsymbol{B}_{i}=\left(0, A r, B_{i z}\right)$ and $\boldsymbol{B}_{e}=\left(0, A a^{2} / r, B_{e z}(a / r)^{2}\right)$, respectively, where $A$ is a constant. In this configuration internal and external magnetic fields are in the same direction and the twist of internal and external fields at the tube/medium boundary is the same (Figure 1). The unperturbed plasma pressure clearly depends on $r$ in order to hold the pressure balance. We use linear ideal incompressible MHD equations, which consist of three components of the momentum equation, three of the induction equation, and the continuity equation. The incompressible approximation considers negligible density perturbations and infinite sound speed. Therefore, the gradient of the pressure perturbation is retained in the momentum 
equation, while the time derivative of density perturbations is neglected in the continuity equation (Chandrasekhar 1961). After straightforward calculations, the total (thermal + magnetic) pressure perturbations are governed by the Bessel equation (Goossens et al. 1992; Zaqarashvili et al. 2014, 2015) which has a solution in terms of Bessel functions. The continuity of the Lagrangian total pressure and displacement results in the dispersion equation

$$
\begin{aligned}
\frac{\left(\left[\omega-k_{z} U\right]^{2}-\omega_{A i}^{2}\right) F_{m}\left(m_{i} a\right)-\frac{2 m A \omega_{A i}}{\sqrt{4 \pi \rho_{i}}}}{\rho_{i}\left(\left[\omega-k_{z} U\right]^{2}-\omega_{A i}^{2}\right)^{2}-\frac{4 A^{2} \omega_{A i}^{2}}{4 \pi}} \\
=\frac{a^{2}\left(\omega^{2}-\omega_{A e}^{2}\right) Q_{\nu}\left(m_{e} a\right)-2 a^{2}\left(\omega^{2}-\omega_{A e}^{2}\right)+\frac{2 m a^{2} A \omega_{A e}}{\sqrt{4 \pi \rho_{e}}}}{a^{2} \rho_{e}\left(\omega^{2}-\omega_{A e}^{2}\right)^{2}-\frac{4 a^{2} A^{2} \omega^{2}}{4 \pi}},
\end{aligned}
$$

where $\omega$ is the angular frequency, $\omega_{A i}$ and $\omega_{A e}$ are the Alfvén frequencies inside and outside the tube respectively, $m$ is the azimuthal wave number, $k_{z}$ is the longitudinal wavenumber, and $\rho_{i}$ and $\rho_{e}$ are the densities inside and outside the tube, respectively. Furthermore,

$$
F_{m}\left(k_{i} a\right)=\frac{k_{i} a I_{m}^{\prime}\left(k_{i} a\right)}{I_{m}\left(k_{i} a\right)}, Q_{\nu}\left(k_{e} a\right)=\frac{k_{e} a K_{\nu}^{\prime}\left(k_{e} a\right)}{K_{\nu}\left(k_{e} a\right)}
$$

where $I_{m}$ and $K_{\nu}$ are modified Bessel functions of order $m$ and $\nu$, respectively, and

$$
\begin{aligned}
& k_{i}^{2}=k_{z}^{2}\left[1-\frac{4 A^{2} \omega_{A i}^{2}}{4 \pi \rho_{i}\left[\left(\omega-k_{z} U\right)^{2}-\omega_{A i}^{2}\right]^{2}}\right], \\
& k_{e}^{2}=k_{z}^{2}\left[1-\frac{4 B_{e \phi}^{2} \omega^{2}}{4 \pi \rho_{i}\left[\omega^{2}-\omega_{A i}^{2}\right]^{2} a^{2}}\right]
\end{aligned}
$$

(see Zaqarashvili et al. 2014).

The dispersion relation (1) is a transcendental equation for the complex wave frequency, $\omega$, whose imaginary part indicates an instability process in the system, in particular, the growth rate of unstable harmonics. In this system, plasma flow in the twisted magnetic field can create velocity discontinuities between the surface of the flux tube and the surrounding media, which may trigger the KHI. To simplify Equation (1) we consider perturbations with the wave vector nearly perpendicular to the magnetic field, i.e., $\boldsymbol{k} \cdot \boldsymbol{B}_{e}=$ $\boldsymbol{k} \cdot \boldsymbol{B}_{i} \approx 0$ which are vortices in the incompressible limit and have the strongest growth rate. In the cylindrical coordinate system, this condition is expressed as

$$
|m| \approx \frac{k_{z} a}{\xi}
$$

where $\xi=a A / B_{i z}$ is the ratio of azimuthal and tangential components of magnetic field inside the tube. Furthermore, we assume that the azimuthal component (twist) of the magnetic field at the boundary of the flux tube is small $(a A \ll 1)$. These assumptions reduce Equation (1) to the form:

$$
\begin{aligned}
& \rho_{i} Q_{\nu}\left(k_{e} a\right) \omega^{2}-2 \rho_{i} k_{z} U Q_{\nu}\left(k_{e} a\right) \omega+\rho_{i} k_{z}^{2} U^{2} Q_{\nu}\left(k_{e} a\right) \\
& \quad-2 \rho_{i} \omega^{2}+4 \rho_{i} k_{z} U \omega-2 \rho_{i} k_{z}^{2} U^{2}-\rho_{e} F_{m}\left(k_{i} a\right) \omega^{2}=0,
\end{aligned}
$$

where $\nu \approx \sqrt{4+m^{2}}$ (see Zaqarashvili et al. 2014 for details). Equation (3) is solved analytically for large azimuthal wave numbers $m$.

For large azimuthal wave numbers $m$ and $\nu$, asymptotic expansion of the modified Bessel functions for large order can be implemented in Equation (3) (see, e.g., Abramowitz \& Stegun 1965). This gives $F_{m}\left(k_{i} a\right) \approx 1, Q_{\nu}\left(k_{e} a\right) \approx-1$ and Equation (3) can be simplified to

$$
\left[1+\frac{1}{3} \frac{\rho_{e}}{\rho_{i}}\right] \omega^{2}-2 k_{z} U \omega+k_{z}^{2} U^{2}=0 .
$$

The solution of Equation (4) is always a complex number, which indicates that the perturbations are unstable to KHI for large azimuthal wave numbers. The growth rate of the unstable harmonics is an imaginary part of the solution of Equation (4),

$$
\omega_{i}=\frac{\sqrt{\rho_{e} / 3 \rho_{i}}}{1+\rho_{e} / 3 \rho_{i}} \frac{|m| \xi}{a} U .
$$

Equation (5) shows that the growth rate of KHI depends on the flow speed, the radius of the tube, the density contrast, the ratio of azimuthal and tangential components of the magnetic field, and the spatial scale of perturbations in the azimuthal direction $(m)$. It can be used to estimate KHI growth times of specific jets in the solar chromosphere.

We note that the dispersion equation similar to Equation (4) for the twisted magnetic flux tube has been solved analytically by Zaqarashvili et al. (2014). They showed that the harmonics with small azimuthal wavenumbers have smaller growth rates compared to those with larger ones. Therefore, we use the large azimuthal wavenumber limit in Equation (3) to consider the unstable harmonics with larger growth rates. There are two additional reasons why the harmonics of large azimuthal wavenumbers are important. First, numerical modeling shows that the photospheric/chromospheric magnetic flux tubes are developing KHI with large azimuthal wavenumbers $(m>5)$ (see e.g., Terradas et al. 2008; Antolin et al. 2014, 2015; Murawski et al. 2016). Second, a large azimuthal wavenumber obviously yields a smaller wavelength, which is important for plasma heating (see Section 4.2).

We note that the incompressibility limit we have used in this model is a valid approximation as compressibility does not have a strong influence on the dynamics of KHI for the harmonics considered here. Soler et al. (2012) has shown that the effect of compressibility on KHI is controlled by the ratio of longitudinal and tangential components of wave vector $\left(k_{y} / k_{z}\right)$ with respect to the magnetic field. An equilibrium magnetic field is straight and directed along $z$ in the configuration used by Soler et al. (2012). For large $k_{y} / k_{z}$, the growth rates of KHI for compressible and incompressible cases are almost similar (see Figure 1 of Soler et al. 2012). For $k_{y} / k_{z} \rightarrow \infty$, which are harmonics propagating perpendicular to the magnetic field, both compressible and incompressible results agree. Therefore, compressibility plays no role when the wave vector is perpendicular to the magnetic field/flow direction. Wentzel (1979) also noted that the perturbations with $k_{z} / k_{y} \ll 1$, where the magnetic field is directed along $z$, involve negligible compression. In our model we have considered harmonics with wave vector perpendicular to the magnetic field (pure vortices) and hence incompressibility is a valid approximation.

Furthermore, our MHD model is developed in the singlefluid approach, where ion-electron and neutral gases are not 
considered as separate fluids. However, for timescales longer than the ion-neutral collision time the single-fluid model is valid as collisions between neutrals and charged particles lead to rapid coupling of the two fluids (see details in Zaqarashvili et al. 2011b). Soler et al. (2015) proposed that in the upper chromosphere, where the ion-neutral collision frequency could be smaller than the ion-ion and ion-electron collision frequencies, the two-fluid model is a better approximation, and showed that this model is valid under these circumstances. However, for spicular jets the ion-neutral collision frequancy is $10^{3} \mathrm{~Hz}$. The corresponding spatial scales are around $50 \mathrm{~m}$ for the chromospheric Alfvén speed $\left(\sim 50 \mathrm{~km} \mathrm{~s}^{-1}\right)$. While we deal with spatial scales of $>50 \mathrm{~km}$ the single-fluid approach is a valid approximation.

\section{OBSERVATIONS AND DATA REDUCTION}

The data presented here were partly studied in Kuridze et al. (2015a). Observations were undertaken between 09:06 and 09:35 UT on 2013 May 3 at disk center with the CRisp Imaging SpectroPolarimeter (CRISP; Scharmer 2006; Scharmer et al. 2008) instrument, mounted on the Swedish $1 \mathrm{~m}$ Solar Telescope (SST; Scharmer et al. 2003a) in La Palma. Adaptive optics were used throughout the observations, consisting of a tip-tilt mirror and a 85-electrode deformable mirror setup that is an upgrade of the system described in Scharmer et al. (2003b). The observations comprised spectral imaging in the H $\alpha 6563 \AA$, and Fe I $6302 \AA$ lines. All data were reconstructed with Multi-Object Multi-Frame Blind Deconvolution (MOMFBD; van Noort et al. 2005). We applied the CRISP data reduction pipeline as described in de la Cruz Rodríguez et al. (2015), including small-scale seeing compensation as in Henriques (2012). Our spatial sampling was 0.!0592 pixel $^{-1}$ and the spatial resolution approached the diffraction limit of the telescope at this wavelength $\left(\sim 0^{\prime \prime} 16\right)$ for a large and steady portion of the images throughout the time series. The $\mathrm{H} \alpha$ line scan consisted of seven positions $(-0.906,-0.543,-0.362$, $0.000,0.362,0.543,+0.906 \AA$ from line core), corresponding to a range of -41 to $+41 \mathrm{~km} \mathrm{~s}^{-1}$ in velocity. A full spectral scan had an acquisition time of $1.3 \mathrm{~s}$, which was also the temporal cadence of the $\mathrm{H} \alpha$ time series. We made use of CRISPEX (Vissers \& Rouppe van der Voort 2012), a versatile widget-based tool for effective viewing and exploration of multi-dimensional imaging spectroscopy data.

\section{ANALYSIS AND RESULTS}

Kuridze et al. (2015a) studied the dynamics of REs in the dataset presented here. They detected a total of 70 RREs in the far red wing at $\mathrm{H} \alpha+0.906 \AA$ and 58 RBEs in the far blue wing at $\mathrm{H} \alpha-0.906 \AA$, and analyzed their lifetime, length, width, speed of their apparent motion, line-of-sight (LOS) velocity, and transverse velocity for each individual detection. A statistical study of their properties showed that the lifetimes of the RBEs/RREs ranged from 10 to $70 \mathrm{~s}$, with a median of 40 $\mathrm{s}$, the lengths are in range $2-9 \mathrm{Mm}$ with a median $\sim 3 \mathrm{Mm}$ and the widths between the 150 and $500 \mathrm{~km}$, with a median of $\sim 260 \mathrm{~km}$ (see Figures 2 and 4 in Kuridze et al. 2015a).

The detected structures also display non-periodic, transverse motions perpendicular to their axes at speeds of $4-31 \mathrm{~km} \mathrm{~s}^{-1}$. Kuridze et al. (2015a) proposed that the transverse motions of chromospheric flux tubes can develop KHI at the tube boundaries due to the velocity discontinuities between the

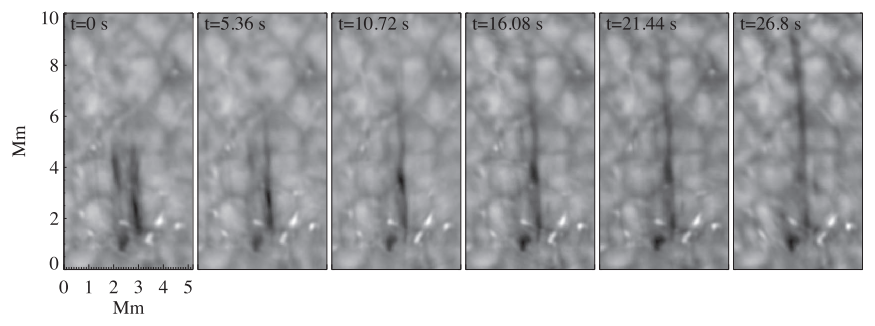

Figure 2. Time sequence of a typical jet in the red wing of $\mathrm{H} \alpha+0.906 \AA\left(41 \mathrm{~km} \mathrm{~s}^{-1}\right)$. The jet starts from the network brightening and moves upward with an apparent propagation speed of $\sim 150 \mathrm{~km} \mathrm{~s}^{-1}$.

surface of the flux tube and surrounding media. Using a simple slab model they showed that the REs moving in the transverse direction with speed comparable to the local Alfvén speed could be unstable due to the KHI with a very short instability growth time (see Kuridze et al. 2015a for further details).

Many of the detected structures appear as high-speed jets that are directed outward from a magnetic bright point. Figure 2 shows the temporal evolution of a typical jet detected in the red wing at $\mathrm{H} \alpha+0.906 \AA$. The jet starts near the bright point and moves upward with an apparent propagation speed of $\sim 150 \mathrm{~km}$ $\mathrm{s}^{-1}$. Apparent velocities of the REs projected on the image plane are in the range $50-150 \mathrm{~km} \mathrm{~s}^{-1}$ (see Figure 4 in Kuridze et al. 2015a) which are Alfvénic and super-Alfvénic in the chromosphere.

Some recent observations show that REs and type II spicules display torsional/twisting motions during their evolution (De Pontieu et al. 2012, 2014). If we consider the chromospheric jets as cylindrical, twisted magnetic flux tubes moving along their axis with respect to the external twisted field, then (as we show in Section 2) these structures may be unstable to KHI.

The growth time, $T$, of the KHI is given by $T=2 \pi / \omega_{i}$, where $\omega_{i}$ is defined by Equation (5). If the KHI is a viable mechanism for the heating/disappearance of REs then the growth time for the instability should be comparable to or smaller than the structure's lifetime. To estimate the dependence of the growth time of the KHI on the azimuthal wave number, we employ three different values of the measured apparent speeds, $U=50,100$, and $150 \mathrm{~km} \mathrm{~s}^{-1}$, and the median value of the radius of REs, $a=130 \mathrm{~km}$, in Equation (5). The results are presented in Figure 3, which represents the growth times derived from Equation (5) which is the solution of the dispersion equation for the high order of $m$. Unfortunately, the present observations do not allow a determination of the density ratios between the outside and the inside of the jet. However, as spicular jets are observed as overdense features (e. g., Beckers 1968), values of growth time are computed for three different density ratios $\rho_{e} / \rho_{i}=0.1,0.5$, and 0.05 . For the ratio of azimuthal and tangential components of magnetic field at the tube boundary in Equation (5), we took $\xi=0.1$ which implies that the magnetic field is only slightly twisted. We see that the the instability growth times strongly depend on the flow speed and density ratio. Higher flow speeds produce faster growth, and higher density ratios slow the growth of the instability (Figure 3). The flow speed, $U \sim 150 \mathrm{~km} \mathrm{~s}^{-1}$, for the harmonics with azimuthal mode numbers $5 \leqslant m \leqslant 40$ leads to growth times of $T \sim 5-85 \mathrm{~s}$, comparable to the lifetimes of REs $(\sim 10-100 \mathrm{~s})$. For speeds in the range $U \sim 50-100 \mathrm{~km} \mathrm{~s}^{-1}$, the KHI growth times for the same harmonics are longer $(T \sim 45-250 \mathrm{~s})$ The higher harmonics have very short instability growth times. For harmonics with $m \geqslant 50$ the 


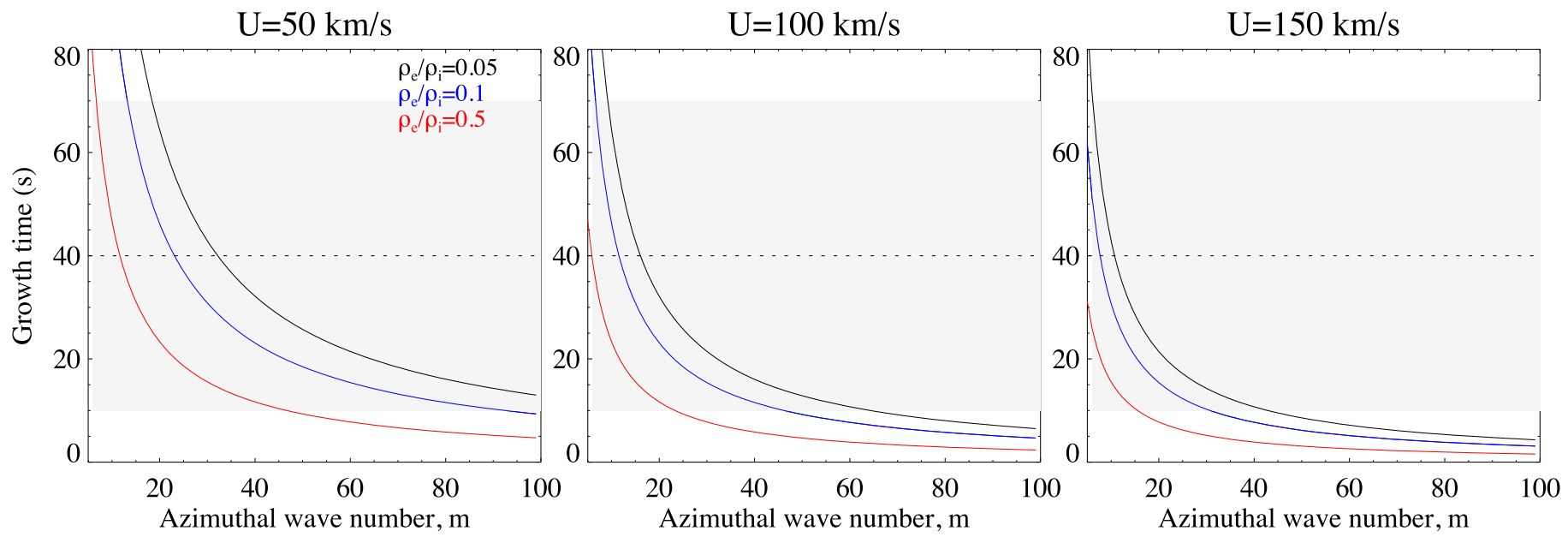

Figure 3. KHI growth times as a function of $m$ for the twisted jets with different density ratios, $\rho_{e} / \rho_{e}$, and flow speed $U$. The growth times are derived from Equation (5) which is the solution of the dispersion equation for the high order of $m$. The ratio of azimuthal and tangential components of magnetic field at the tube boundary is $\xi=0.1$. The gray-shaded areas indicate the typical range of lifetimes $(\sim 10-70 \mathrm{~s})$ with the horizontal dotted lines indicating the average lifetimes of REs.
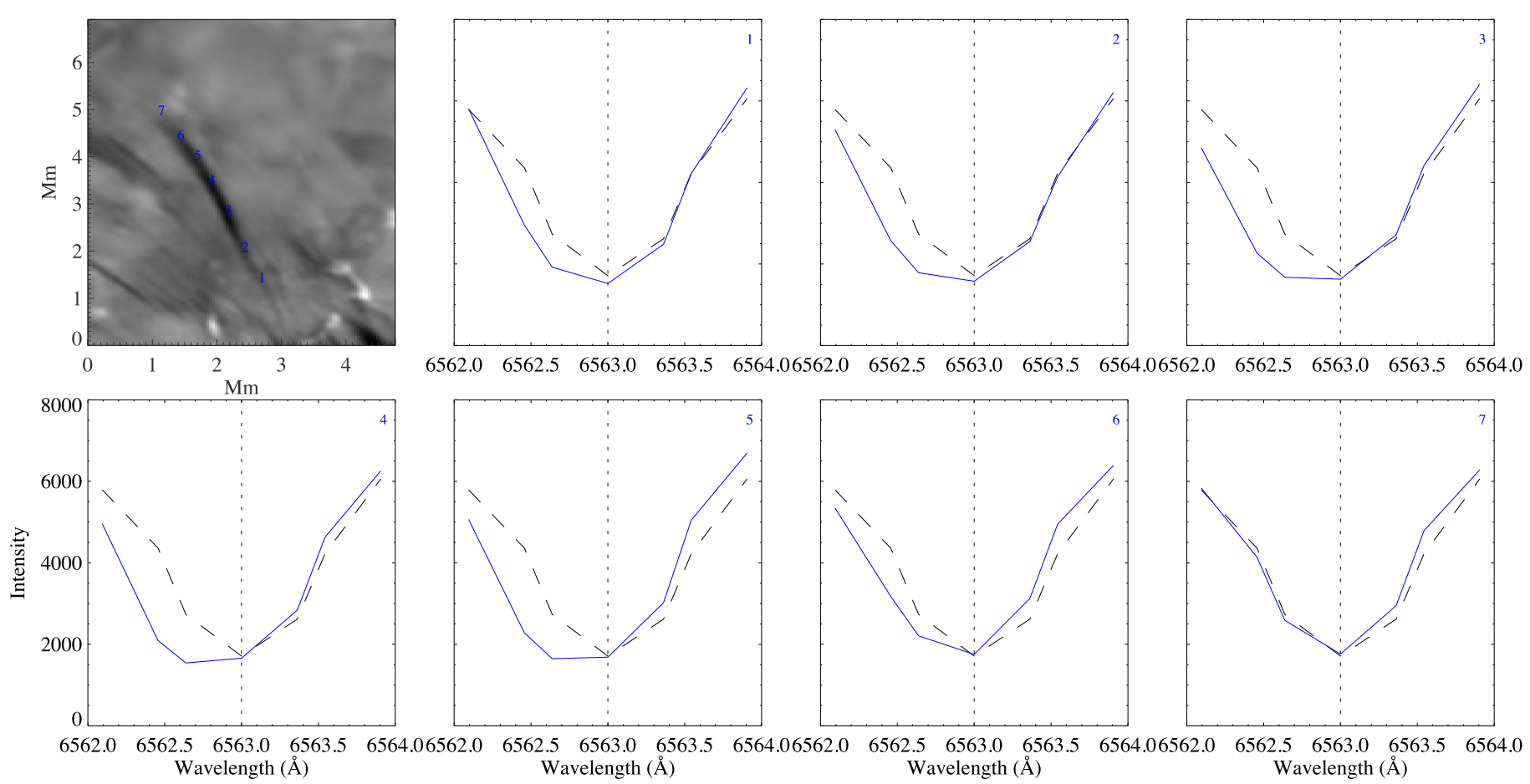

Figure 4. $\mathrm{H} \alpha$ line profiles (blue lines) of typical RBEs (detected in the $\mathrm{H} \alpha+0.906 \AA$ ) at different positions along their lengths. Numbers indicate the locations of line profiles. The black lines show the mean spectrum average over the field of view for reference.

growth times are $1-5 \mathrm{~s}$ depending on the flow speed and density ratio.

The generated $\mathrm{KH}$ vortices may lead to enhanced MHD turbulence near the boundaries of the REs, which could be observed as non-thermal broadening in the spectral line profiles. We therefore study the evolution of the $\mathrm{H} \alpha$ line profiles for the detected REs. Figure 4 represents the line profiles of typical RBEs at different positions along their length (blue lines) together with a mean spectrum average over the field of view for reference (black lines). We see that the line profiles have an extended absorption wing on the blue side of the core, and they are wider all along the structures' lengths (Figure 4). The line profile at position 7, which is from outside of the structure, is very close to the mean profile. Values of Doppler width for each individual feature are calculated from
Doppler signals provided by their $\mathrm{H} \alpha$ spectral profile using the second moment with respect to wavelength method described by Rouppe van der Voort et al. (2009). The Doppler width of the structures is computed for every pixel and every frame of the REs. Full paths of the REs were selected manually, but the computations were only performed where the wing showed an opacity dip according to the criteria described in Rouppe van der Voort et al. (2009). The computed Doppler width for each individual detection is shown in Figure 5, where the final values for each pixel, detected as valid, are used. Figure 5 indicates that the Doppler widths increase for the REs, with average width for RBEs and RREs found to be $\sim 10 \mathrm{~km} \mathrm{~s}^{-1}$ and $\sim 12 \mathrm{~km} \mathrm{~s}^{-1}$, respectively. It should be noted that these estimates do not have a large accuracy as the method employs the line spectrum to separate and compute the LOS Doppler 


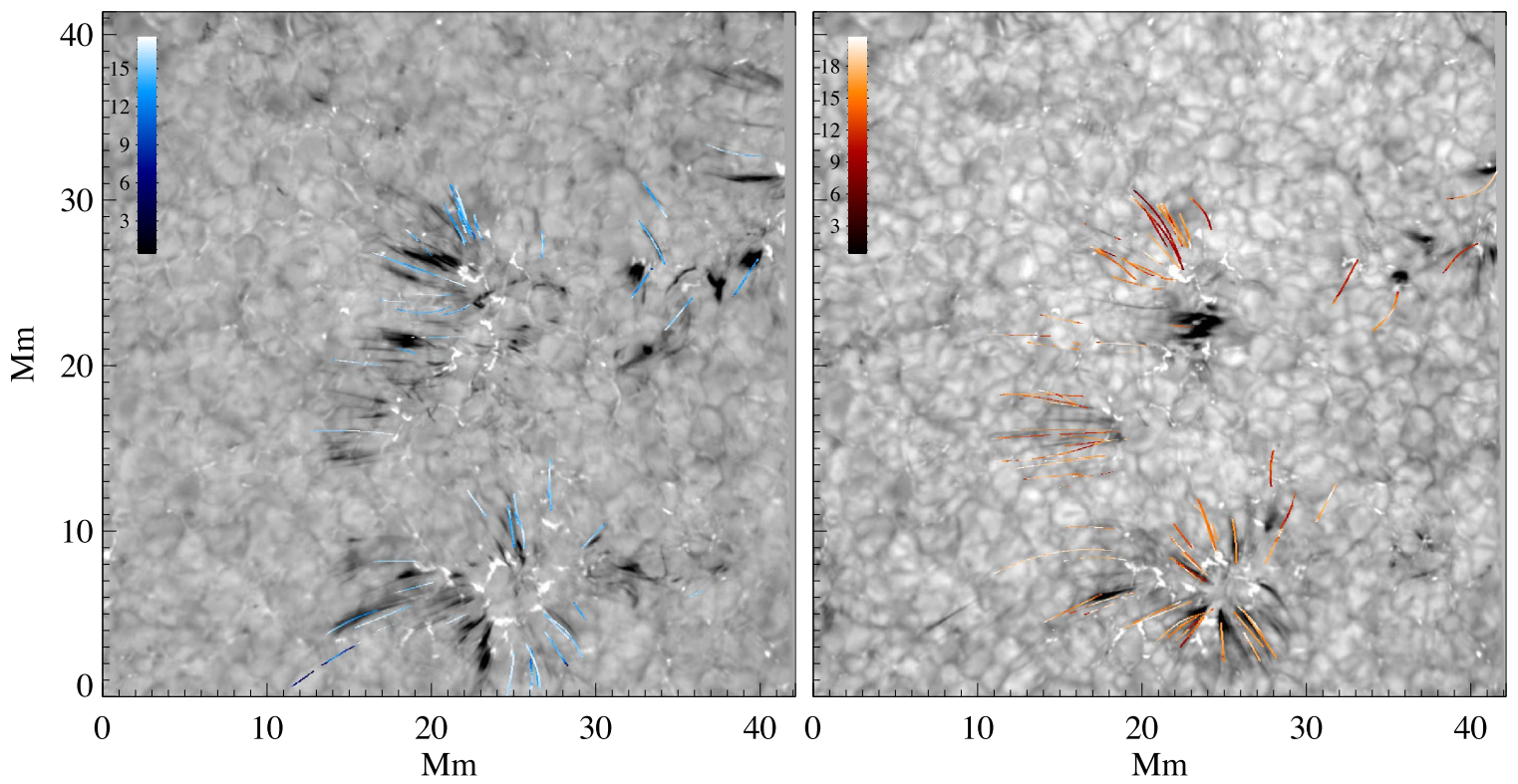

Figure 5. H $\alpha$ Doppler widths along RBEs (left panel) and RREs (right panel) over-plotted at their locations in the field of view, with the color scale indicating the velocity in $\mathrm{km} \mathrm{s}^{-1}$.

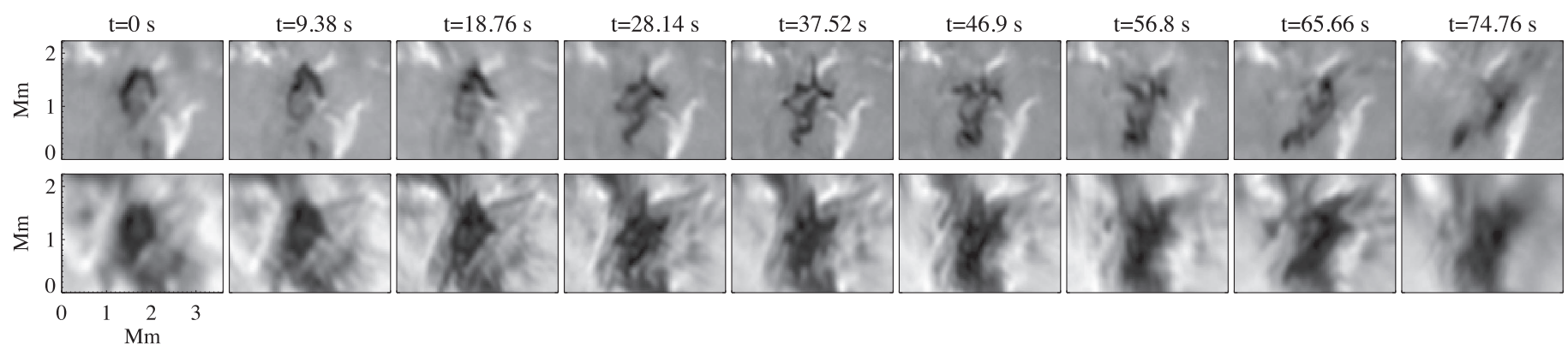

Figure 6. Sequence of frames showing the temporal evolution of the jet ejected along the LOS in $\mathrm{H} \alpha-0.906 \AA$ (top) and $\mathrm{H} \alpha-0.543 \AA$ of $\mathrm{H} \alpha$ (bottom) images. Initially the jet has a circular top; however, it rapidly develops azimuthal nodes around its boundary.

velocity and Doppler width components using first and second moments of the profile. Another limitation of the method is that it computes the line width for only the blue or red component of the line wing, as normally there is no spectral signature of the detection at the opposite side of the wing. Nonetheless, the analysis shows clearly that the REs detected in red and blue wings in $\mathrm{H} \alpha$ have enhanced line widths all along the structures' lengths (Figure 5). This suggest that, if line broadening is produced by turbulent motions associated with KHI vortices, then the longitudinal scale of the KHI should be much shorter than the length of the structures.

The wavelength of the KHI perturbation can be determined using $\lambda=2 \pi / k_{z}$, where $k_{z} \approx|m| \xi / a$ is defined from Equation (2). It shows that the harmonics with high order of azimuthal wavenumbers, which have faster growth time, correspond to KHI perturbations with short wavelengths. For $a=130, \xi=0.1$ and $30 \leqslant|m| \leqslant 100$, the wavelength is in the range $\lambda \simeq 70-270 \mathrm{~km}$, much less than the RE measured lengths $(\sim 3.5 \mathrm{Mm})$.

It should be also noted that the line broadening can be caused by rotation/torsional motion of the structures around the axis. This may happen when the spatial resolution of observations is less than the width of the structures, and one cannot resolve the opposite directed motions at the left and right sides of the structures. The spatial resolution of the presented $\mathrm{H} \alpha$ observations is around half $(\sim 120 \mathrm{~km})$ of the measured average width $(\sim 260 \mathrm{~km})$ of REs, and hence the rotational motion should be resolved. However, we have not detected this in the current dataset, which suggests that MHD turbulence/heating is the ultimate source of line broadening.

As well as small-scale REs, larger-scale jets have also been observed near the center of the rosettes in the blue wing of $\mathrm{H} \alpha$, and an example is shown in Figure 6. It appears in the far blue wings at $-0.906 \AA$ and $-543 \AA$ from the $\mathrm{H} \alpha$ line core with speed of $\sim 34 \mathrm{~km} \mathrm{~s}^{-1}$ and width $\sim 800 \mathrm{~km}$. The structure does not have a horizontal extension, indicating that the jet was ejected vertically to the solar surface along the LOS (Figure 6). At the start of the ejection the jet has a circular-shaped top. However, the structure rapidly develops small vortex-shaped features on timescales of tens of seconds near its boundary (Figure 6). These features may correspond to the projected vortex-flows of the KHI perturbations. Unfortunately, $\sim 75 \mathrm{~s}$ after the jet's first appearance, the observation stopped and we were unable to study the full lifecycle of the jet. However, in the last images the jet almost disappeared from the far blue wing image at $\mathrm{H} \alpha-0.906 \AA$ (Figure 6), indicating that it was in the disappearing phase of its life. The growth time of the $\mathrm{KHI}$ as a function of azimuthal wave number, $m$, obtained from Equation (7) for the jet with radius, $a=400 \mathrm{~km}$, upflow speed $U=34 \mathrm{~km} \mathrm{~s}^{-1}$, and the ratio of azimuthal and tangential 


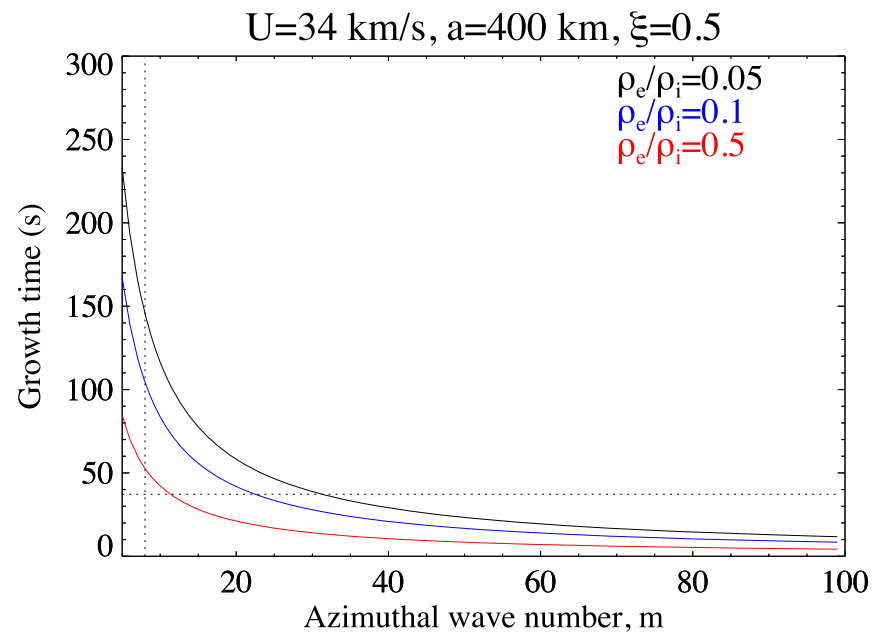

Figure 7. KHI growth times as a function of $m$ for the large-scale twisted jets. The horizontal and vertical dotted lines indicate an vortex formation time $(\sim 37 \mathrm{~s})$, and the number of azimuthal nodes $(m \approx 8)$, respectively, estimated from Figure 6

components of magnetic field $\xi=0.5$, are presented in Figure 7. It can be estimated that at around $t=37.52 \mathrm{~s}$, the erupting structure has around eight resolved projected vortexlike features/azimuthal nodes around its boundary (see Figure 6). For the harmonic $m=8$ the growth time $T \sim 52-145 \mathrm{~s}$, depending on the values of the density ratio outside and inside the tube (Figure 7). This growth time appears to be consistent with the timescale $(\sim 37 \mathrm{~s})$ taken for the structure to develop vortex-like features (Figure 6). $\xi=0.5$ is well below the threshold for kink instability, which starts around $\xi=2$, therefore the dispersion equation is still correct for such a twist. However, for lower $\xi$, i.e., $\xi=0.1$ the growth time is much longer, $T \sim 260-700 \mathrm{~s}$, depending on the values of the density ratio.

\subsection{Heating Signatures in the TR and Corona}

Recent observations have revealed that chromospheric type II spicules are rapidly heated to $\mathrm{TR}$ and even coronal temperatures. Using the dataset presented here, Henriques et al. (2016) showed the connection between REs and coronal structures. The automated detections of coronal transients was achieved by employing a running difference technique, aiming to have the highest sensitivity for $50 \mathrm{~s}$ transients. By using a robust statistical analysis based on Chernoff bounds, absolute minimum values of $6 \%$ of the SDO/AIA $171 \AA$ channel events and $11 \%$ of the $304 \AA$ events were shown to have an RE counterpart. The probability of the observed matches being due to noise or random chance was demonstrated to be lower than $10^{-40}$. Figure 8 shows some specific examples, where the structures are clearly seen in all channels including the RE maps, $304 \AA$ and $171 \AA$ lines (for more examples see Figures 3-9 and the associated time series from Henriques et al. 2016). Such particular matches were also found by Pereira et al. (2014) between $304 \AA$ and type II spicules in the $H_{\alpha}$ line, and by Rouppe van der Voort et al. (2015) in the TR channels.

\subsection{Heating of Chromospheric Jets Due to KHI}

The rapid heating of chromospheric jets is an unsolved problem. An initial explanation is that KHI destroys the jets by the rapid mixture of chromospheric and coronal plasmas, which

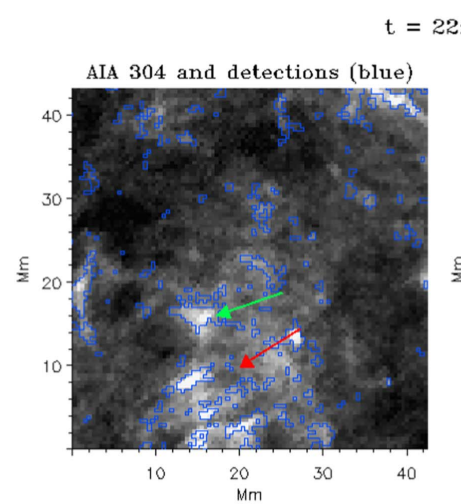

$t=22: 41$
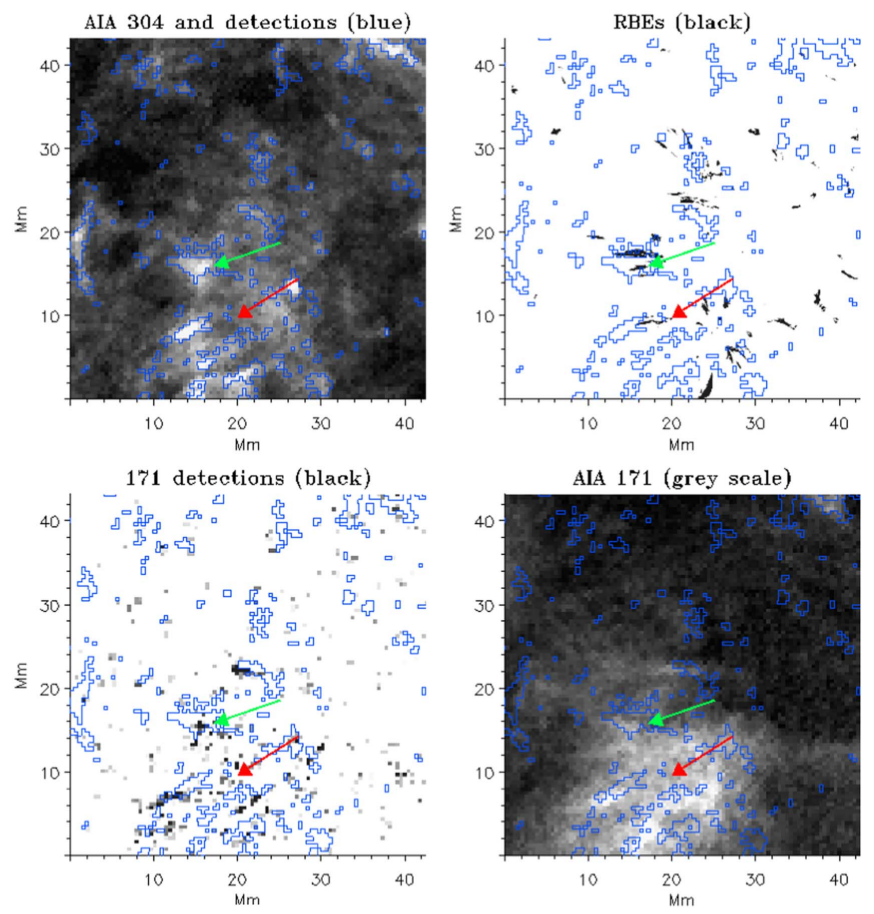

Figure 8. Panels extracted from the first time-series of Henriques et al. (2016) where we highlight two examples of jets crossing the chromosphere and the corona (one color per jet). The arrows are directed at the same point across all channels: the far-right signature of each jet which is visible as a tip from highcontrast RBEs (see panel labelling). The thin dark path traced by the red-arrow $\mathrm{RBE}$ is seen connecting with an elongated blue contour. This blue contour comes from an automated detection algorithm sensitive to $50 \mathrm{~s}$ variations in the AIA $304 \AA$ channel. However, for this frame, such a variation is also visible as a bright patch in the AIA $304 \AA$ intensity (top left panel). This contour connects, at the other end of the elongation, with a similarly detected $171 \AA$ channel signature (which is also visible in the $171 \AA$ intensity). This indicates a phased progression across all channels, which most certainly involves changes in temperature and likely ionization state, considering the formation ranges involved. The green arrow jet follows the same pattern, with the difference that all signatures are nearly overlapped as their shape is much narrower across all channels (indicating a more vertical propagation), and the $304 \AA$ detection contour encompasses the RBE, the 171 detection, the 171 brightening, and another co-propagating RBE.

could lead to the rapid disappearance of the jets. However, in this case the jets must be destroyed completely (as indicated by numerical simulations) and should not appear in hotter spectral lines, in clear contrast with observations. On the other hand, large-scale $\mathrm{KH}$ vortices may transfer their energy into smaller scales through a nonlinear cascade, where it could be transformed into heat. Furthermore, several dissipation mechanisms can produce a direct energy transfer from $\mathrm{KH}$ vortices into heat without a non-linear cascade.

In our simplified ideal MHD model the various dissipation effects, such as diffusivity, viscosity, radiative cooling, and thermal conduction, are neglected. These MHD processes could have an important impact on the lifetime and stability of chromospheric structures. Unfortunately, the exact effects of these dissipation processes are not well studied in spicule-like jets. However, the lifetimes of the small-scale REs are too short to be explained by these dissipation processes. Lipartito et al. (2014) estimated that only structures that have a radial scale less than $5 \mathrm{~km}$ are expected to fail the frozen field condition and diffuse within their lifetime $(\sim 100 \mathrm{~s})$. This suggests that the time taken for chromospheric jets with a typical width of 
$\sim 250 \mathrm{~km}$ to diffuse is expected to be much longer than their thermodynamical lifetime.

As spicular jets are chromospheric features, thermal conduction probably has a small effect on their dynamics over such short timescales $(\sim 100 \mathrm{~s})$. The heating/cooling timescale due to thermal conduction can be written as

$$
\tau_{c} \approx \frac{3 n k_{\mathrm{B}} L^{2}}{2 / 7 \kappa T^{5 / 2}}
$$

where $\kappa=1.1 \times 10^{-6} \mathrm{erg} \mathrm{s}^{-1} \mathrm{~cm}^{-1} \mathrm{~K}^{-1}$ is the Spitzer thermal conductivity, $k_{\mathrm{B}}$ the Boltzmann constant, $T$ the temperature, and $n$ and $L$ the number density and half-length of the structure, respectively (Cargill \& Klimchuk 1997; Tsiropoula \& Tziotziou 2004). For the typical number density of spicular jets, $\mathrm{n} \sim 10^{11} \mathrm{~cm}^{-3}$, half-length $\sim 1.5 \mathrm{Mm}$ and TR temperature $\sim 10^{5} \mathrm{~K}$, the thermal conduction timescale is around 26 hours, much longer than the lifetime of the observed features.

Tsiropoula \& Tziotziou (2004) have shown that the energy flux due to radiative cooling in chromospheric $\mathrm{H} \alpha$ mottles is much higher than the conductive flux. Typical radiative cooling times are expected to be of the order of minutes (Anderson \& Athay 1989; Judge 2006), still longer than the dynamical timescales of REs.

It should be noted that in the theoretical model we have assumed a fully ionized plasma. However, the chromosphere is only partially ionized, with the ratio of neutral atoms to electrons continuously increasing from almost zero at the transition region to $\sim 10^{4}$ at the photospheric base. The interaction of ions and neutral atoms influences basic plasma processes leading to the damping of MHD waves and/or electric currents (Song \& Vasyliūnas 2011, 2014; Khomenko \& Collados 2012). Both types of spicules, as well as RREs/ RBEs, have to be composed of partially ionized plasmas, and therefore ion-neutral collisions may lead to heating of the $\mathrm{KH}$ vortices and consequently the structure itself. However, a detailed study of the heating process is not the main goal of the present paper, and the characteristic heating times of different dissipation mechanisms can be estimated through the energy equation.

The equation of energy in a partially ionized plasma can be written as (see e.g., Zaqarashvili et al. 2011a)

$$
\begin{aligned}
\frac{\partial p}{\partial t} & +(\boldsymbol{V} \cdot \nabla) p+\gamma p \nabla \cdot \boldsymbol{V} \\
= & (\gamma-1) \frac{\alpha_{\mathrm{ei}}}{e^{2} n_{e}^{2}} \boldsymbol{j}^{2}+(\gamma-1) \alpha_{\mathrm{in}} \boldsymbol{w}^{2} \\
& +(\gamma-1) Q_{\mathrm{visc}}+Q_{\mathrm{cool}},
\end{aligned}
$$

where $p$ and $\boldsymbol{V}$ are the total pressure and the velocity of protons, electrons, and neutral hydrogen atoms respectively, $\alpha_{\mathrm{ei}}$ and $\alpha_{\text {in }}$ the coefficients of friction between ions and electrons, and ions and neutral hydrogen atoms, respectively, $n_{e}$ the electron number density, $\boldsymbol{j}=(c / 4 \pi) \nabla \times \boldsymbol{B}$ the current, $\boldsymbol{w}=\boldsymbol{V}_{i}-\boldsymbol{V}_{n} \approx$ $\left(\xi_{n} / \boldsymbol{c} \alpha_{\text {in }}\right) \boldsymbol{j} \times \boldsymbol{B}$ the velocity difference between protons and neutrals, $\xi_{n}$ the ratio of neutral to total particle density, $\gamma=5 / 3$ the ratio of specific heats, and $c$ the speed of light. The quantity $Q_{\text {visc }}=-\pi_{\alpha \beta} W_{\alpha \beta}$ is the heating due to viscosity, where $\pi_{\alpha \beta}$ is the viscous stress tensor and $W_{\alpha \beta}$ the shear velocity tensor (Braginskii 1965). On the right-hand side of Equation (6), the first, second, and third terms are associated with heating by Joule, ion-neutral collision, and viscosity, respectively. The last term,
$Q_{\text {cool }}$, is associated with several cooling processes such as thermal conduction and radiation. However, as discussed above, these processes are unimportant over the timescales of REs and hence may be ignored. The ratio of the first term (associated with Joule heating) to the second (heating by ion-neutral collisions) on the right-hand side of Equation (6) is approximately

$$
\frac{Q_{\mathrm{ei}}}{Q_{\mathrm{in}}} \approx \frac{m_{i} m_{e} c^{2} \delta_{\mathrm{ei}} \delta_{\mathrm{in}}}{e^{2} \xi_{n} B^{2}},
$$

where $m_{i}$ and $m_{e}$ are the proton and electron masses, respectively, $\delta_{\mathrm{ei}}$ and $\delta_{\text {in }}$ the electron-ion and ion-neutral collision frequencies, respectively, $e$ is the electron charge, and $B$ is the magnetic field strength.

It must be noted that the ion-neutral collision frequency $\delta_{\text {in }}$ is generally different from that for neutral-ion collisions $\delta_{\mathrm{ni}}$ in partially ionized plasmas. They differ by the factor of ionization fraction. Zaqarashvili et al. (2011b) suggested that the actual physical meaning of the collision time is expressed by the formula $1 /\left(\delta_{\text {in }}+\delta_{\text {ni }}\right)$, which shows the timescale over which the relative velocity between ions and neutrals $\left(\boldsymbol{w}=\boldsymbol{V}_{i}-\boldsymbol{V}_{n}\right)$ decreases exponentially. Hence, it shows the timescale of energy exchange between ions and neutrals. This suggestion was fully verified by recent numerical simulations (Oliver et al. 2016). Unfortunately, observations do not allow us to estimate the precise ionization fraction in chromospheric jets. However, we know that the ionization fraction is changing from almost zero at the TR $\left(T \sim 10^{5} \mathrm{~K}\right)$ to $\sim 1$ at the photospheric base $\left(T \sim 5 \times 10^{3} \mathrm{~K}\right)$. Based on some parameters given by the cloud model, Tsiropoula \& Schmieder (1997) derived an ionization degree for hydrogen in chromospheric jets (mottles) of around 0.65. Verth et al. (2011) also estimated the ionization degree of hydrogen atoms along a spicule. They showed that the lower part of the spicule has a small ionization degree (0.01-0.1), but the upper half has a value close to 0.5 . Therefore, in the following we adopt an ionization degree of $\sim 0.5$, which yields the same values of ionneutral and neutral-ion collision frequencies. From Soler et al. (2015), at a height of $2000 \mathrm{~km}$ and for $\xi_{n}=0.5, \delta_{\mathrm{ei}}=10^{7} \mathrm{~Hz}$ and $\delta_{\mathrm{in}}=\delta_{\mathrm{ni}}=10^{3} \mathrm{~Hz}$. For the magnetic field strength of $B=10 \mathrm{G}$, the ratio $Q_{\mathrm{ei}} / Q_{\text {in }}$ is approximately 0.0023 , which means that the heating due to ion-neutral collisions is much stronger than the Ohmic heating in chromospheric jets.

Khodachenko et al. (2004) showed that viscosity effects are much smaller those of ion-neutral collisions in the solar chromosphere. Indeed, the ratio of the third (associated to viscous heating) and the second terms on the right-hand side of Equation (8) is approximately

$$
\frac{Q_{\mathrm{visc}}}{Q_{\mathrm{in}}} \sim \frac{V^{2} \beta}{V_{A}^{2}} \frac{\delta_{\mathrm{in}} \delta_{i i}}{\nu_{\mathrm{ci}}^{2}},
$$

where $\beta=8 \pi p / B^{2}$ is the plasma beta parameter, $\delta_{i i}$ the ion-ion collision frequency, $\omega_{\mathrm{ci}}$ the ion gyrofrequency, $V$ the plasma velocity, and $V_{A}$ the Alfvén speed. For an ion number density of $10^{11} \mathrm{~cm}^{-3}$ and temperature of $10^{4} \mathrm{~K}$, typical for chromospheric jets, the ion-ion collision frequency is $\delta_{i i} \approx 10^{5} \mathrm{~Hz}$. A velocity of $V=10 \mathrm{~km} \mathrm{~s}^{-1}$, magnetic field strength of $10 \mathrm{G}$, ion gyrofrequency $\nu_{\mathrm{ci}}=10^{5} \mathrm{~Hz}$, typical chromospheric Alfvén speed of $50 \mathrm{~km} \mathrm{~s}^{-1}, \delta_{\text {in }}=10^{3} \mathrm{~Hz}$ and plasma beta $\beta=0.1$, gives a value for the ratio of viscous to ion-neutral heating 
$Q_{\text {visc }} / Q_{\text {in }} \sim 10^{-5}$. Therefore, viscous heating is negligible compared to that by ion-neutral collisions.

These estimates suggest that ion-neutral collisions (the second term on right-hand side of Equation (8)) define the heating time of $\mathrm{KH}$ vortices. The heating time for certain harmonics can be written as

$$
t_{\text {heat }} \sim \frac{\beta \delta_{\text {in }} D^{2}}{V_{A}^{2}} \frac{1-\xi_{n}}{\xi_{n}^{2}},
$$

where $D$ is the characteristic width of a $\mathrm{KH}$ vortex. If the jet radius is $a=130 \mathrm{~km}$, then the characteristic widths of $m=20-50$ harmonics are $D=2 \pi a / m \approx 16-40 \mathrm{~km}$. Furthermore, the typical Alfvén speed of $50 \mathrm{~km} \mathrm{~s}^{-1}, \xi_{n}=0.5$, $\delta_{\text {in }}=10^{3} \mathrm{~Hz}$ and plasma beta $\beta=0.1$ yield a heating time of $\sim 20-130$ s. This simple estimation shows that the KH vortices may heat the plasma over timescales which are comparable to the lifetime of chromospheric jets.

\section{DISCUSSION AND CONCLUSION}

Some recent theoretical and observational studies suggest that $\mathrm{H} \alpha$ jets, observed ubiquitously in the quiet-Sun chromosphere, could be unstable to KHI due to the presence of velocity discontinuities between the surface of the jet and the surrounding plasma (Zaqarashvili 2011; Zaqarashvili et al. 2015). Chromospheric jets, observed normally near network boundaries rooted in regions with photospheric magnetic bright points, are traditionally interpreted as overdense magnetic flux tubes with field aligned flows. However, it is suggested that at least some of the energetic, short-lived, type II spicular features may correspond to warps in two-dimensional sheets instead of flux tubes (Judge et al. 2011, 2012). Chromospheric jets also exhibit transverse and torsional motions during their lifetime (De Pontieu et al. 2012, 2014). The latter can produce a magnetic twist in the flux tubes where structures are formed.

To investigate theoretical aspects of the stability of chromospheric jets, we employ the KHI theory for the twisted jets developed in the incompressible ideal MHD limit. The chromospheric jets are modeled as cylindrical, high-density, twisted magnetic flux tubes moving in an axial direction in the twisted magnetized environment. We derive dispersion equations governing the dynamics of twisted jets and solve them analytically in the large azimuthal wavelength limit. The solutions of the dispersion equations indicate that the perturbations with large azimuthal wave number $m$ are unstable to KHI with any upflow speed, $U$. From the imaginary part of the solution and measured jet parameters we estimate the growth time of KHI (Figure 3). It shows that for REs, the growth times for $5 \leqslant m \leqslant 40$ are between 5 and $250 \mathrm{~s}$, and for $m \geqslant 40$ the growth times are 1-35 s, depending on the flow speed and density ratio.

The turbulent motion and non-thermal heating produced by KHI can be observed as a broadening of the spectral profiles of chromospheric jets. We employ $\mathrm{H} \alpha$ spectra of the detected features to compute their Doppler widths. The analysis shows that the widths of the $\mathrm{H} \alpha$ line profiles are broadened with respect to the neighboring areas (Figures 4, 5). Similar findings have been reported in previous studies (Cauzzi et al. 2009; Rouppe van der Voort et al. 2009; Sekse et al. 2013a; Lipartito et al. 2014). The analyzed REs have enhanced line widths all along the structures' lengths, indicating that if the line broadening is produced by turbulent motions associated with KHI vortices, then the longitudinal scale of the KHI should be much shorter than the length of the structures. Indeed, the estimated wavelengths of the KHI perturbation $(\lambda \sim 70-270 \mathrm{~km})$ are much less than the REs' measured lengths $(\sim 3.5 \mathrm{Mm})$.

It must be noted that the approach used here to compute the Doppler width has certain limitations, as it attributes the changes in the absorption line profiles intensities to the line width and LOS velocities only, while intensity changes are in general proportional to density, wavelength-dependent opacity, and the source function. Recently, Kuridze et al. (2015b) has shown that the intensity changes in the $\mathrm{H} \alpha$ line profile are highly dependent on the velocity gradient in the solar chromosphere, as it can create differences in the opacity between the red and blue wings of the line core. Furthermore, the $\mathrm{H} \alpha$ line positions in our observations had a maximum step of $0.363 \AA$ which generates an uncertainty in our measurements of the Doppler parameters of the order of $7 \mathrm{~km} \mathrm{~s}^{-1}$. Advanced cloud modeling with chromospheric radiative transfer calculations, together with observations at improved spectral resolution, would allow us to compute velocities and line widths for the chromospheric jets with higher accuracy.

Direct observational evidence of KHI perturbations in REs would be the detections of vortex-like features/azimuthal nodes at their boundaries. The widths of the REs analyzed in this paper are only about twice as large as the spatial resolution at $\mathrm{H} \alpha$, which leaves the KHI vortices/azimuthal nodes unresolved in the current dataset. However, as well as small-scale REs, we have detected a larger $\mathrm{H} \alpha$ jet, which appears as a circular-shaped absorption feature in the blue wing of $\mathrm{H} \alpha$ (Figure 6). Its radius $(\sim 400 \mathrm{~km})$ and LOS velocity $\left(\sim 34 \mathrm{~km} \mathrm{~s}^{-1}\right)$ suggest that this jet could be the on-disk counterpart of a macrospicule or $\mathrm{H} \alpha$ surge frequently observed at the solar limb. The morphology of the jet suggests that the plasma flow was oriented along the LOS. Figure 6 shows that the structure develops azimuthal nodes over timescales of tens of seconds around its boundary. From a visual inspection we estimate $m \sim 8$ projected vortex-likes flows at around $t=37.52 \mathrm{~s}$ (Figure 6). Translating this number of observed azimuthal nodes into growth times using our theoretical model, we obtain $T \sim 52-145 \mathrm{~s}$ for the density ratios of $0.5-0.05$, which appears to be consistent with the timescale $(\sim 37 \mathrm{~s})$ for the structure to develop the nodes in the image sequence presented in Figure 6.

KHI can produce fast heating of chromospheric jets if the large-scale KHI vortices are decomposed through a nonlinear cascade of energy transfer to small scales. Furthermore, there are several damping mechanisms that could be responsible for a direct energy transfer of large-scale KHI vortices into heat without a nonlinear cascade to small scales. In the theoretical model presented in Section 2 we used an adiabatic approximation in an ideal regime for a fully ionized plasma, which neglects heating processes and non-ideal effects such as diffusivity, viscosity, radiative cooling, thermal conduction, and Ohmic dissipation. Therefore, the model presented cannot reproduce heating as a direct effect of KHI. We have shown that the heating timescales associated with diffusivity, thermal conduction and radiative losses are much longer than the dynamic timescales of observed chromospheric jets. To investigate further nonlinear, non-adiabatic effects we provide estimates of characteristic heating times for other dissipation processes through the analysis of the energy equation in the partially ionized plasma. The results indicate that ion-neutral 
collisions could be the most important process for the heating of the $\mathrm{KH}$ vortices and consequently the structure itself. For REs we estimate timescales of the heating due to the ionneutral collisions, for KHI vortices with a high order of azimuthal wavenumbers, to be $\sim 20-130 \mathrm{~s}$, comparable to the lifetimes of these chromospheric jets. We note that a similar approach was used in the classical work of Braginskii (1965), where MHD wave equations were solved without neutrals and the results were used in an energy equation with neutrals to study the damping of MHD waves and heating rate due to the ion-neutral collisions. Such an approach allows a preliminary study of the possible KHI-associated heating in the cylindrical, twisted magnetic jets, for which there is no direct analytical solution when including partially ionized plasma in the basic MHD model presented in Section 2.

Due to the striking match between observed timescales and those of ion-neutral heating at KHI vortices, further assessement of the importance of KHI to the heating of chromospheric jets, via detailed numerical simulations/forward modeling, is recommended. Furthermore, higher temporal, spatial and spectral resolution observations (e.g., GREGOR, SST, DKIST, BBSO) will provide a better opportunity to resolve and study the KHI perturbations in chromospheric fine-scale structures.

The research leading to these results has received funding from the European Community's Seventh Framework Programme (FP7/2007-2013) under grant agreement no. 606862 (F-CHROMA). The work of T.V.Z. was supported by the Austrian "Fonds zur Förderung der wissenschaftlichen Forschung under projects P26181-N27 and P25640-N27, and by FP7-PEOPLE-2010-IRSES-269299 project- SOLSPANET.

\section{REFERENCES}

Abramowitz, M., \& Stegun, I. A. 1965, Handbook of Mathematical Functions with Formulas, Graphs, and Mathematical Tables (New York: Dover)

Anderson, L. S., \& Athay, R. G. 1989, ApJ, 346, 1010

Antolin, P., Okamoto, T. J., De Pontieu, B., et al. 2015, ApJ, 809, 72

Antolin, P., Yokoyama, T., \& Van Doorsselaere, T. 2014, ApJL, 787, L22

Beckers, J. M. 1968, SoPh, 3, 367

Berger, T. E., Slater, G., Hurlburt, N., et al. 2010, ApJ, 716, 1288

Braginskii, S. I. 1965, RvPP, 1, 205

Browning, P. K., \& Priest, E. R. 1984, A\&A, 131, 283

Cargill, P. J., \& Klimchuk, J. A. 1997, ApJ, 478, 799

Cauzzi, G., Reardon, K., Rutten, R. J., Tritschler, A., \& Uitenbroek, H. 2009, A\&A, 503, 577

Chandrasekhar, S. 1961, Hydrodynamic and Hydromagnetic Stability (Oxford: Clarendon)

de la Cruz Rodríguez, J., Löfdahl, M. G., Sütterlin, P., Hillberg, T., \& Rouppe van der Voort, L. 2015, A\&A, 573, A40

De Pontieu, B., Carlsson, M., Rouppe van der Voort, L. H. M., et al. 2012, ApJL, 752, L12

De Pontieu, B., Erdélyi, R., \& James, S. P. 2004, Natur, 430, 536

de Pontieu, B., McIntosh, S., Hansteen, V. H., et al. 2007, PASJ, 59, 655

De Pontieu, B., McIntosh, S. W., Carlsson, M., et al. 2011, Sci, 331, 55

De Pontieu, B., Rouppe van der Voort, L., McIntosh, S. W., et al. 2014, Sci, 346,1255732

Feng, L., Inhester, B., \& Gan, W. Q. 2013, ApJ, 774, 141

Foullon, C., Verwichte, E., Nakariakov, V. M., Nykyri, K., \& Farrugia, C. J. 2011, ApJL, 729, L8

Foullon, C., Verwichte, E., Nykyri, K., Aschwanden, M. J., \& Hannah, I. G. 2013, ApJ, 767, 170

Goossens, M., Andries, J., \& Arregui, I. 2006, RSPTA, 364, 433

Goossens, M., Andries, J., \& Aschwanden, M. J. 2002, A\&A, 394, L39

Goossens, M., Erdélyi, R., \& Ruderman, M. S. 2011, SSRv, 158, 289

Goossens, M., Hollweg, J. V., \& Sakurai, T. 1992, SoPh, 138, 233

Henriques, V. M. J. 2012, A\&A, 548, A114

Henriques, V. M. J., Kuridze, D., Mathioudakis, M., \& Keenan, F. P. 2016, ApJ, 820, 124
Hollweg, J. V. 1981, SoPh, 70, 25

Hollweg, J. V. 1982, ApJ, 257, 345

Judge, P. 2006, in ASP Conf. Ser. 354, Solar MHD Theory and Observations A High Spatial Resolution Perspective, ed. J. Leibacher, R. F. Stein, \& H. Uitenbroek (San Francisco, CA: ASP), 259

Judge, P. G., Reardon, K., \& Cauzzi, G. 2012, ApJL, 755, L11

Judge, P. G., Tritschler, A., \& Chye Low, B. 2011, ApJL, 730, L4

Khodachenko, M. L., Arber, T. D., Rucker, H. O., \& Hanslmeier, A. 2004, A\&A, 422, 1073

Khomenko, E., \& Collados, M. 2012, ApJ, 747, 87

Kuridze, D., Henriques, V., Mathioudakis, M., et al. 2015a, ApJ, 802, 26

Kuridze, D., Mathioudakis, M., Simões, P. J. A., et al. 2015b, ApJ, 813, 125 Langangen, Ø., De Pontieu, B., Carlsson, M., et al. 2008, ApJL, 679, L167

Lipartito, I., Judge, P. G., Reardon, K., \& Cauzzi, G. 2014, ApJ, 785, 109

Madjarska, M. S., Vanninathan, K., \& Doyle, J. G. 2011, A\&A, 532, L1

Martínez-Gómez, D., Soler, R., \& Terradas, J. 2015, A\&A, 578, A104

Möstl, U. V., Temmer, M., \& Veronig, A. M. 2013, ApJL, 766, L12

Murawski, K., Chmielewski, P., Zaqarashvili, T. V., \& Khomenko, E. 2016, MNRAS, 459, 2566

Murawski, K., \& Zaqarashvili, T. V. 2010, A\&A, 519, A8

Ofman, L., Davila, J. M., \& Steinolfson, R. S. 1994, GeoRL, 21, 2259

Ofman, L., \& Thompson, B. J. 2011, ApJL, 734, L11

Oliver, R., Soler, R., Terradas, J., \& Zaqarashvili, T. V. 2016, ApJ, 818, 128

Pataraya, A. D., Taktakishvili, A. L., \& Chargeishvili, B. B. 1990, SoPh, 128,333

Pereira, T. M. D., De Pontieu, B., Carlsson, M., et al. 2014, ApJL, 792, L15

Roberts, B. 1979, SoPh, 61, 23

Rouppe van der Voort, L., De Pontieu, B., Pereira, T. M. D., Carlsson, M., \& Hansteen, V. 2015, ApJL, 799, L3

Rouppe van der Voort, L., Leenaarts, J., de Pontieu, B., Carlsson, M., \& Vissers, G. 2009, ApJ, 705, 272

Ryutova, M., Berger, T., Frank, Z., Tarbell, T., \& Title, A. 2010, SoPh, 267, 75

Scharmer, G. B. 2006, A\&A, 447, 1111

Scharmer, G. B., Bjelksjo, K., Korhonen, T. K., Lindberg, B., \& Petterson, B. 2003a, Proc. SPIE, 4853, 341

Scharmer, G. B., Dettori, P. M., Lofdahl, M. G., \& Shand, M. 2003b, Proc. SPIE, 4853, 370

Scharmer, G. B., Narayan, G., Hillberg, T., et al. 2008, ApJL, 689, L69

Sekse, D. H., Rouppe van der Voort, L., \& De Pontieu, B. 2013a, ApJ, 764,164

Sekse, D. H., Rouppe van der Voort, L., De Pontieu, B., \& Scullion, E. 2013b, ApJ, 769, 44

Soler, R., Ballester, J. L., \& Zaqarashvili, T. V. 2015, A\&A, 573, A79

Soler, R., Díaz, A. J., Ballester, J. L., \& Goossens, M. 2012, ApJ, 749, 163

Soler, R., Terradas, J., Oliver, R., Ballester, J. L., \& Goossens, M. 2010, ApJ, 712,875

Song, P., \& Vasyliūnas, V. M. 2011, JGRA, 116, A09104

Song, P., \& Vasyliūnas, V. M. 2014, ApJL, 796, L23

Suematsu, Y., Shibata, K., Neshikawa, T., \& Kitai, R. 1982, SoPh, 75, 99

Terradas, J., Andries, J., Goossens, M., et al. 2008, ApJL, 687, L115

Tian, H., DeLuca, E. E., Cranmer, S. R., et al. 2014, Sci, 346, 1255711

Tsiropoula, G., \& Schmieder, B. 1997, A\&A, 324, 1183

Tsiropoula, G., \& Tziotziou, K. 2004, A\&A, 424, 279

Tsiropoula, G., Tziotziou, K., Kontogiannis, I., et al. 2012, SSRv, 169, 181

Uchida, Y. 1969, PASJ, 21, 128

van Noort, M., Rouppe van der Voort, L., \& Löfdahl, M. G. 2005, SoPh, 228, 191

Vanninathan, K., Madjarska, M. S., Scullion, E., \& Doyle, J. G. 2012, SoPh, 280,425

Verth, G., Goossens, M., \& He, J.-S. 2011, ApJL, 733, L15

Vissers, G., \& Rouppe van der Voort, L. 2012, ApJ, 750, 22

Wentzel, D. G. 1979, ApJ, 227, 319

Zaqarashvili, T. V. 2011, in AIP Conf. Ser. 1356, 3rd School and Workshop on Plasma Physics, ed. I. Zhelyazkov \& T. Mishonov (Melville, NY: AIP), 106

Zaqarashvili, T. V., Díaz, A. J., Oliver, R., \& Ballester, J. L. 2010, A\&A, 516, A84

Zaqarashvili, T. V., Khodachenko, M. L., \& Rucker, H. O. 2011a, A\&A, 534, A93

Zaqarashvili, T. V., Khodachenko, M. L., \& Rucker, H. O. 2011b, A\&A, 529, A82

Zaqarashvili, T. V., Vörös, Z., \& Zhelyazkov, I. 2014, A\&A, 561, A62

Zaqarashvili, T. V., Zhelyazkov, I., \& Ofman, L. 2015, ApJ, 813, 123

Zhang, Y. Z., Shibata, K., Wang, J. X., et al. 2012, ApJ, 750, 16

Zhelyazkov, I. 2012, A\&A, 537, A124

Zhelyazkov, I., \& Zaqarashvili, T. V. 2012, A\&A, 547, A14 